ANNALES

POLONICI MATHEMATICI

$81.1(2003)$

\title{
Approximation results for nonlinear integral operators in modular spaces and applications
}

\author{
by Ilaria Mantellini and Gianluca Vinti (Perugia)
}

\begin{abstract}
We obtain modular convergence theorems in modular spaces for nets of operators of the form $\left(T_{w} f\right)(s)=\int_{H} K_{w}\left(s-h_{w}(t), f\left(h_{w}(t)\right)\right) d \mu_{H}(t), w>0, s \in G$, where $G$ and $H$ are topological groups and $\left\{h_{w}\right\}_{w>0}$ is a family of homeomorphisms $h_{w}: H \rightarrow h_{w}(H) \subset G$. Such operators contain, in particular, a nonlinear version of the generalized sampling operators, which have many applications in the theory of signal processing.
\end{abstract}

1. Introduction. The starting point of this paper comes directly from the applications in the theory of signal processing.

The famous Whittaker-Kotelnikov-Shannon (WKS) sampling theorem, formulated in the last century, states that, given a function $f \in L^{2}(\mathbb{R})$ which is band-limited in an interval $[-\pi w, \pi w]$ for $w>0$, it is possible to reconstruct $f$ on the whole real axis by means of an interpolation formula. The interpolation used takes into account only the behaviour of the function $f$ in its sample values $f(k / w)$ calculated at the nodes $k / w$, for $k \in \mathbb{Z}$, uniformly spaced on the whole real axis, and gives

$$
f(t)=\sum_{k=-\infty}^{\infty} f(k / w) \operatorname{sinc}[\pi(w t-k)], \quad t \in \mathbb{R} .
$$

This theorem can be formulated in the language of information transmission theory (see [10]) in the following manner: let $f$ be a signal of finite energy on $\mathbb{R}$ and of bounded frequency spectrum contained in $[-\pi w, \pi w]$, which means that this signal does not contain frequencies higher than $w / 2$ cycles per second. Moreover let the signal have a certain communication channel. In order to recover this signal at the output of this communication channel it is sufficient to transmit over this channel only the values $f(k / w)$ of the signal at the nodes $k / w$.

2000 Mathematics Subject Classification: 41A35, 46A80, 47G10, $47 \mathrm{H} 30$.

Key words and phrases: nonlinear integral operators, modular spaces, modular convergence, nonlinear generalized sampling series. 
Here $1 / w$ is the so called Nyquist "interval" corresponding to the frequency domain $[-\pi w, \pi w]$, which is the theoretical minimum time interval between the samples in order to reconstruct the signal completely (see e.g. [20]).

The interpolation formula (I), which allows one to reconstruct the signal $f$, in practice has some disadvantages. Indeed, according to (I), in order to reconstruct the signal completely, one should know the behaviour of $f$ in an infinite number of sample values, which one usually does not have at one's disposal. Moreover, if $t$ represents the present time, then formula (I) says that one should know the samples of the signal not only in the past of $t$, but also in the future, i.e. for $k / w>t$. Still more, in the WKS-sampling theorem, the signal should be band-limited, which implies that it is not duration limited; and the latter is the class of signals which occurs in practice.

In order to overcome the above disadvantages, the idea of P. L. Butzer and his school was to replace the sinc function in (I) by a function $\varphi$ which is continuous with compact support contained in an interval $\left[T_{0}, T_{1}\right]$. Obviously in this manner, the behaviour of the function $\varphi$ is such that it is sufficient to know only a finite number of sample values and, if $0<T_{0}<T_{1}$, then the sample values can be taken only from the past, which means to make a prediction of the signal; furthermore the signal need not be band-limited. Of course one should not expect a formula like (I), but an approximation result for $f$ can be obtained.

Namely, in [16] a family of operators, called generalized sampling operators, is introduced, of the form

$$
\left(S_{w}^{\varphi} f\right)(t)=\sum_{k=-\infty}^{\infty} f(k / w) \varphi(w t-k), \quad t \in \mathbb{R}, k \in \mathbb{Z}, w>0,
$$

and a theorem on pointwise and uniform convergence of $S_{w}^{\varphi} f$ toward $f$ as $w \rightarrow \infty$ is proved.

In [8] a nonlinear version of the generalized sampling operators is introduced and a uniform convergence result is proved which extends the Butzer-Stens result. Moreover the study of nonlinear approximation in signal processing is important since the approximation describes a nonlinear system in which the computed signal, during its filtering, generates new frequencies.

But it would be of some interest to formulate such approximation results not only for uniformly continuous functions (and hence with respect to uniform convergence), but also for functions belonging to an $L^{p}$ space, or more generally to some function space. In [28] an approximation result is proved by means of a family of nonlinear generalized sampling operators for func- 
tions belonging to Orlicz spaces; such spaces extend the classical $L^{p}$-spaces. In fact, in [28] a general family of nonlinear integral operators is considered, which contains the generalized sampling operators; in this way, a unified approach has been furnished to the study of convergence theorems for several classes of nonlinear integral operators, well known in the literature. These results extend previous results for linear integral operators given in [7].

In this paper, we study the problem of approximating a function $f$ belonging to a general modular space (in particular, an Orlicz space, a Musielak-Orlicz space and many others) by means of a family of nonlinear integral operators of the form

$$
\left(T_{w} f\right)(s)=\int_{H} K_{w}\left(s-h_{w}(t), f\left(h_{w}(t)\right)\right) d \mu_{H}(t), \quad w>0,
$$

for $s \in G$, where $G$ and $H$ are topological groups; here $\left\{h_{w}\right\}_{w>0}$ is a family of homeomorphisms $h_{w}: H \rightarrow h_{w}(H) \subset G, f: G \rightarrow \mathbb{R}$ is a function belonging to $X(G)$, the space of all Borel measurable real-valued functions defined on $G$, and $K_{w}: G \times \mathbb{R} \rightarrow \mathbb{R}, w>0$, is a net of kernels satisfying suitable conditions.

The main result of the paper is an approximation theorem for the above family of operators when the function $f$ belongs to a modular space (Theorem 4). In these spaces it is possible to introduce a concept of convergence, called "modular convergence", which is more natural in this setting and "weaker" than the usual norm convergence, i.e. the convergence induced by the Luxemburg norm (the theory of modular spaces can be found in $[23,21,26])$. Namely for the operators (II) we prove that

$$
\lim _{w \rightarrow \infty} \varrho_{G}\left[c\left(T_{w} f-f\right)\right]=0
$$

for some $c>0$, where $\varrho_{G}$ is the modular generating the modular space $L_{\varrho}(G)$.

The general setting of modular spaces has required the introduction of some conditions which are satisfied when the modular, generating the modular space, has an integral representation; this happens, for example, in $L^{p}$-spaces, in Orlicz spaces and in Musielak-Orlicz spaces.

The class of integral operators (II) is very general and covers, as particular cases, the classical convolution integral operators, Mellin convolution operators and generalized sampling operators in their nonlinear form (see Section 4). Hence our theory furnishes several applications to the above mentioned classes of nonlinear integral operators. Moreover, the general setting of locally compact topological groups in which we work permits us to recover also the case of the multidimensional generalized sampling series of $f$ (see [12] for the linear case), which has, like the one-dimensional series, many applications in the theory of signal processing. 
2. Notations and definitions. Let $G$ and $H$ be locally compact and $\sigma$-compact Hausdorff topological groups and let $\mu_{G}$ and $\mu_{H}$ be their Haar measures on the classes of Borel sets $\mathcal{B}(G)$ and $\mathcal{B}(H)$ respectively. We will assume that $G$ and $H$ are abelian, but unimodularity is sufficient for our theory. We remark that this condition is automatically satisfied for compact groups (see $[19,17]$ ). We will denote by $\mathcal{U}$ the neighbourhood base of the neutral element $\theta \in G$, and by local compactness, we can use a base with measurable, symmetric, compact neighbourhoods of $\theta$.

We denote by $X(G)$ the vector space of all Borel measurable real-valued functions defined on $G$ and by $C_{\mathrm{c}}(G)$ the subspace of all continuous functions with compact support. For $G=\left(\mathbb{R}^{n},+\right)$ we denote by $C_{\mathrm{c}}^{(k)}\left(\mathbb{R}^{n}\right)$ the space of all real-valued functions with compact support and with continuous derivatives of order $k$, for $1 \leq k \leq \infty$.

Let now $\left\{h_{w}\right\}_{w>0}$ be a net of functions $h_{w}: H \rightarrow G$ such that $h_{w}$ is a homeomorphism between $H$ and $h_{w}(H)$, for every $w>0$.

Let $Z=\left(Z, \Sigma_{Z}, \mu_{Z}\right)$ be an arbitrary measure space and let $X(Z)$ be the corresponding vector space of all $\Sigma_{Z}$-measurable real-valued functions on $Z$. A functional $\varrho: X(Z) \rightarrow[0,+\infty]$ is said to be a modular on $X(Z)$ if the following conditions hold:

(i) $\varrho(f)=0 \Leftrightarrow f=0 \mu_{Z}$-a.e. in $Z$;

(ii) $\varrho(-f)=\varrho(f)$ for every $f \in X(Z)$;

(iii) $\varrho(\alpha f+\beta g) \leq \varrho(f)+\varrho(g)$ for every $f, g \in X(Z)$ and $\alpha, \beta \in \mathbb{R}_{0}^{+}$with $\alpha+\beta=1$.

The functional $\varrho$ generates the modular space $L_{\varrho}$ defined as follows:

$$
L_{\varrho} \equiv L_{\varrho}(Z)=\left\{f \in X(Z): \lim _{\lambda \rightarrow 0} \varrho(\lambda f)=0\right\} .
$$

It is well known that $L_{\varrho}$ is a vector subspace of $X(Z)$ and it is possible to define on it the concept of modular convergence as follows: we say that a sequence of functions $f_{n} \in L_{\varrho}$ is modular convergent (or $\varrho$-convergent) to a function $f \in L_{\varrho}$ if there exists a $\lambda>0$ such that

$$
\lim _{n \rightarrow \infty} \varrho\left(\lambda\left(f_{n}-f\right)\right)=0 .
$$

The modular convergence is weaker than the convergence induced by the Luxemburg norm generated by the modular $\varrho$ (see $[25,23])$. The latter is equivalent to saying that the above limit relation is satisfied for any $\lambda>0$.

The two convergences become equivalent in the space of finite elements defined by

$$
E_{\varrho} \equiv E_{\varrho}(Z)=\{f \in X(Z): \varrho(\lambda f)<\infty \text { for every } \lambda>0\} .
$$

We will need the following definitions concerning modular functionals. We remark that for modulars having an integral representation, the conditions below are always satisfied. 
We say that a modular $\varrho$ is:

(a) monotone if $\varrho(f) \leq \varrho(g)$ whenever $|f| \leq|g|$ for every $f, g \in X(Z)$;

(b) finite if the characteristic function $\chi_{A}$ of every measurable set $A$ of finite $\mu_{Z}$-measure belongs to $L_{\varrho}$;

(c) strongly finite if each $\chi_{A}$ as above belongs to $E_{\varrho}$;

(d) absolutely finite if $\varrho$ is finite and if for every $\varepsilon, \lambda_{0}>0$ there exists a $\delta>0$ such that $\varrho\left(\lambda_{0} \chi_{B}\right)<\varepsilon$ for every $B \in \Sigma_{Z}$ with $\mu_{Z}(B)<\delta$;

(e) absolutely continuous if there is an $\alpha>0$ such that for every $f \in$ $X(Z)$ with $\varrho(f)<\infty$, the following two conditions hold:

(i) for every $\varepsilon>0$ there exists a measurable subset $A \subset Z$ with $\mu_{Z}(A)<\infty$ such that $\varrho\left(\alpha f \chi_{Z \backslash A}\right)<\varepsilon$;

(ii) for every $\varepsilon>0$ there exists a $\delta>0$ such that $\varrho\left(\alpha f \chi_{B}\right)<\varepsilon$ for all measurable sets $B \subset Z$ with $\mu_{Z}(B)<\delta$.

For some of the above concepts we refer to $[24,5]$. Note that in case $\mu_{Z}(G)<\infty$, if $\varrho$ is strongly finite and absolutely continuous then it is also absolutely finite (see [4]).

Let now $\left(G, \mathcal{B}(G), \mu_{G}\right)$ and $\left(H, \mathcal{B}(H), \mu_{H}\right)$ be two locally compact and $\sigma$-compact topological groups and let $\left\{\mu_{w}\right\}_{w>0}$ be a family of functions $\mu_{w}: G \times \mathcal{B}(H) \rightarrow \mathbb{R}_{0}^{+}$such that $\mu_{w}(\cdot, A)$ is measurable for every $A \in \mathcal{B}(H)$ and $\mu_{w}(s, \cdot)$ is a measure on $\mathcal{B}(H)$.

We need to introduce a notion of regularity for the family $\left\{\mu_{w}\right\}_{w>0}$. We will say that $\left\{\mu_{w}\right\}_{w>0}$ is regular if:

(a) putting $\mu_{w}^{s}=\mu_{w}(s, \cdot)$ for $w>0, s \in G$ we have $0 \leq \mu_{w}^{s} \ll \mu_{H}$ for every $s \in G, w>0$;

(b) $\sup _{w>0}\left\|\mu_{w}^{(\cdot)}(H)\right\|_{\infty}<\infty$ and there exists a measurable set $F \subset G$ with $\mu_{G}(F)=0$ and two positive constants $r$ and $\bar{w}$ such that $0<r<$ $\mu_{w}^{(s)}(H)$ for every $w>\bar{w}$ and every $s \in G \backslash F$;

(c) if we set $\xi_{w}(s, t)=d \mu_{w}^{s} / d \mu_{H}$, then $\xi_{w}$ is a globally measurable function and $\left\|\xi_{w}(\cdot, t)\right\|_{1} \leq \eta_{w}$ for every $t \in H$ and some $\eta_{w} \in \mathbb{R}^{+}$.

Now, in the general setting of modular spaces, we need to introduce a condition of compatibility between the family $\left\{\mu_{w}\right\}_{w>0}$ and the modulars $\varrho_{G}$ and $\varrho_{H}$.

Let $\varrho_{G}, \varrho_{H}$ be modular functionals on $X(G)$ and $X(H)$ respectively. We will say that a regular net $\left\{\mu_{w}\right\}_{w>0}$ is compatible with the couple $\left(\varrho_{G}, \varrho_{H}\right)$ if there are two constants $D, M>0$ and a net $\left\{b_{w}\right\}$ of positive numbers with $b_{w} \rightarrow 0$ as $w \rightarrow \infty$ such that

$$
\varrho_{G}\left(\int_{H} g(t) d \mu_{w}^{(\cdot)}(t)\right) \leq M \eta_{w} \varrho_{H}(D g)+b_{w}
$$


for any $g \geq 0, g \in X(H)$ and for sufficiently large $w>0$, where $\eta_{w}$ is the net appearing in condition (c) of the definition of regularity of $\left\{\mu_{w}\right\}_{w>0}$.

Using a similar reasoning to Proposition 1 of [22], one can prove that if $\varrho_{G}$ and $\varrho_{H}$ are convex modulars on an Orlicz space, then every regular net $\left\{\mu_{w}\right\}_{w}$ is compatible with the couple $\left(\varrho_{G}, \varrho_{H}\right)$; later on, we will see that, under suitable assumptions, it is possible to find other examples of regular nets $\left\{\mu_{w}\right\}_{w>0}$ compatible with the couple $\left(\varrho_{G}, \varrho_{H}\right)$.

Let now $G$ be a locally compact and $\sigma$-compact topological group. According to Theorem 1 of [22], we have the following result which we will use in the next section:

THEOREM 1. Let @ be a modular on $X(G)$, absolutely continuous, monotone and absolutely finite. Then $\overline{C_{\mathrm{c}}(G)}=L_{\varrho}(G)$ where the bar represents the modular closure.

3. Approximation theorems. Define, for every $U \in \mathcal{U}$ and $w>0$, the sets

$$
U_{s, w}=\left\{t \in H: s-h_{w}(t) \in U\right\}=h_{w}^{-1}(s+U) .
$$

Moreover, let $\left\{L_{w}\right\}_{w>0}$ be a family of measurable functions $L_{w}: G \rightarrow \mathbb{R}_{0}^{+}$ with $L_{w}\left(s-h_{w}(\cdot)\right) \in L_{\mu_{H}}^{1}(H)$ for $s \in G$ and $w>0$. We will say that $\left\{L_{w}\right\}_{w>0} \in \mathcal{L}$ if the following conditions are satisfied:

(L1) the family $L_{w}$ has compact support in the sense that for every $U \in \mathcal{U}$ there is a $\bar{w}>0$ such that for every $w>\bar{w}$, the supports of $L_{w}$ are contained in $U$;

(L2) there is a constant $N>0$ such that

$$
\int_{H} L_{w}\left(s-h_{w}(t)\right) d \mu_{H}(t) \leq N
$$

for every $s \in G$ and every $w>0$;

(L3) there is a measurable set $F \subset G$ with $\mu_{G}(F)=0$ and two positive constants $r$ and $\bar{w}$ such that, for every $w>\bar{w}$ and every $s \in G \backslash F$,

$$
\int_{H} L_{w}\left(s-h_{w}(t)\right) d \mu_{H}(t)>r .
$$

We now consider the class $\Psi$ of all continuous nondecreasing functions $\psi: \mathbb{R}_{0}^{+} \rightarrow \mathbb{R}_{0}^{+}$such that $\psi(0)=0, \psi(u)>0$ for $u>0$ and $\psi(u) \rightarrow \infty$ as $u \rightarrow \infty$.

Let $\left\{K_{w}\right\}_{w>0}$ be a family of measurable functions $K_{w}: G \times \mathbb{R} \rightarrow \mathbb{R}$ and denote by $\mathcal{K}$ the class of all such families satisfying:

$(\mathrm{K} 1) K_{w}\left(s-h_{w}(\cdot), u\right) \in L_{\mu_{H}}^{1}(H)$ for every $u \in \mathbb{R}$ and $K_{w}(s, 0)=0$ for every $s \in G, w>0$; 
(K2) there is a function $\psi \in \Psi$ such that $K_{w}(s, \cdot)$ is $\left(L_{w}, \psi\right)$-Lipschitz, i.e. there are measurable functions $L_{w}: G \rightarrow \mathbb{R}_{0}^{+}$with $\left\{L_{w}\right\}_{w>0} \in \mathcal{L}$ such that

$$
\left|K_{w}(s, u)-K_{w}(s, v)\right| \leq L_{w}(s) \psi(|u-v|)
$$

for every $s \in G, u, v \in \mathbb{R}$ and $w>0$;

(K3) for every $n \in \mathbb{N}$ and $w \in \mathbb{R}^{+}$, putting

$$
r_{n}^{w}(s):=\sup _{1 / n \leq|u| \leq n}\left|\frac{1}{u} \int_{H} K_{w}\left(s-h_{w}(t), u\right) d \mu_{H}(t)-1\right|,
$$

we have $\lim _{w \rightarrow \infty} r_{n}^{w}(s)=0$ a.e. $s \in G$ (see [28]).

REMARK 1. For $\left\{K_{w}\right\}_{w>0} \in \mathcal{K}$, if we suppose that (K3) is satisfied with $\lim _{w \rightarrow \infty} r_{n}^{w}(s)=0$ uniformly with respect to $s \in G$, then assumption (L3) is satisfied uniformly with respect to $s \in G$ (see Lemma 1 of [8]).

Now let $\varrho_{H}$ and $\eta_{H}$ be two modulars on $X(H)$. The following definition which relates $\varrho_{H}$ and $\eta_{H}$ and the function $\psi$ will be of fundamental importance (see [5]):

We say that the triple $\left\{\varrho_{H}, \psi, \eta_{H}\right\}$ is properly directed if for every $\lambda \in$ ]0,1[ there exists a $\left.C_{\lambda} \in\right] 0,1[$ satisfying

$$
\varrho_{H}\left[C_{\lambda} \psi(|F(\cdot)|)\right] \leq \eta_{H}(\lambda F(\cdot)) \quad \text { for all } F \in X(H) .
$$

Finally, for $\left\{K_{w}\right\}_{w>0} \in \mathcal{K}$, we define the operators

$$
\left(T_{w} f\right)(s)=\int_{H} K_{w}\left(s-h_{w}(t), f\left(h_{w}(t)\right)\right) d \mu_{H}(t)
$$

for every $f \in X(G)$ for which $\left(T_{w} f\right)(s)$ is well defined for $s \in G, w>0$ and $\left(T_{w} f\right)(s)$ is measurable on $G$.

We will use the following corollary proved in [7]:

Corollary 1. For every nonempty open set $A \subset G$ there is a $\bar{w}>0$ such that $h_{w}^{-1}(A) \neq \emptyset$ for every $w \geq \bar{w}$.

Now we state the following modular approximation theorem for functions belonging to $C_{\mathrm{c}}(G)$ :

Theorem 2. Let $\left\{K_{w}\right\}_{w>0} \in \mathcal{K}$ and $\left\{L_{w}\right\}_{w>0} \in \mathcal{L}$; let $\varrho$ be a monotone, strongly finite and absolutely continuous modular on $X(G)$. Then for every $f \in C_{\mathrm{c}}(G)$ and $\lambda>0$, we have

$$
\lim _{w \rightarrow \infty} \varrho\left[\lambda\left(T_{w} f-f\right)\right]=0 .
$$

Proof. Let $f \in C_{\mathrm{c}}(G)$ and let $C=\operatorname{supp} f$. We put

$$
C_{w}=\left\{t \in H: h_{w}(t) \in C\right\}=h_{w}^{-1}(C) .
$$


By Corollary $1, C_{w}$ is a nonempty compact subset of $H$ for sufficiently large $w$, and if $t \notin C_{w}$, we have $f\left(h_{w}(t)\right)=0$ by (K1). Therefore

$$
\left(T_{w} f\right)(s)=\int_{C_{w}} K_{w}\left(s-h_{w}(t), f\left(h_{w}(t)\right)\right) d \mu_{H}(t), \quad s \in G .
$$

By (K1) and (K2), we have

$$
\left|K_{w}\left(s-h_{w}(t), f\left(h_{w}(t)\right)\right)\right| \leq L_{w}\left(s-h_{w}(t)\right) \psi\left(\left|f\left(h_{w}(t)\right)\right|\right) .
$$

Now, by (L1), denoting by $U$ a compact neighbourhood of $\theta \in G$ such that $\operatorname{supp} L_{w} \subset U$ for sufficiently large $w>0$, we may deduce that $\operatorname{supp} K_{w}(\cdot, u)$ $\subset U$ for sufficiently large $w>0$ and every $u \in \mathbb{R}$. Put now $B=U+C$. If $s \notin B$, then for every $t \in C_{w}, s-h_{w}(t) \notin U$, and $K_{w}\left(s-h_{w}(t), u\right)=0$ for sufficiently large $w>0$; hence $T_{w} f$ vanishes outside the compact set $B$. Thus $\varrho\left[\lambda\left(T_{w} f-f\right)\right]=\varrho\left[\lambda\left(T_{w} f-f\right) \chi_{B}\right]$. By the $\left(L_{w}, \psi\right)$-Lipschitz condition,

$$
\begin{aligned}
\left|\left(T_{w} f\right)(s)-f(s)\right| & \leq\left|\left(T_{w} f\right)(s)\right|+|f(s)| \\
& =\left|\int_{H} K_{w}\left(s-h_{w}(t), f\left(h_{w}(t)\right)\right) d \mu_{H}(t)\right|+|f(s)| \\
& \leq \int_{H} L_{w}\left(s-h_{w}(t)\right) \psi\left(\left|f\left(h_{w}(t)\right)\right|\right) d \mu_{H}(t)+|f(s)| \\
& \leq N \psi\left(\|f\|_{\infty}\right)+\|f\|_{\infty}
\end{aligned}
$$

for $s \in G$ and $w>0$. So

$$
\lambda\left|T_{w} f(s)-f(s)\right| \chi_{B} \leq \lambda \chi_{B}\left[N \psi\left(\|f\|_{\infty}\right)+\|f\|_{\infty}\right]
$$

for $s \in G$. Since the modular is strongly finite, we have $\chi_{B} \in E_{\varrho}$ and therefore

$$
\varrho\left[\lambda\left(T_{w} f-f\right) \chi_{B}\right] \leq \varrho\left[\lambda\left(N \psi\left(\|f\|_{\infty}\right)+\|f\|_{\infty}\right) \chi_{B}\right]<\infty .
$$

By Proposition 1 of [28] it is possible to prove Theorem 1 of [8] with the almost everywhere convergence of $r_{n}^{w}(s)$ (assumption (K3)) obtaining $\left(\left(T_{w} f\right)(s)-f(s)\right) \rightarrow 0$ as $w \rightarrow \infty$ a.e. $s \in G$. Hence, applying the Lebesgue dominated convergence theorem for modular spaces (see [24]), we obtain

$$
\lim _{w \rightarrow \infty} \varrho\left[\lambda\left(T_{w} f-f\right)\right]=0
$$

Let now $\mathcal{L}^{*} \subset \mathcal{L}$ be the subclass of $\mathcal{L}$ whose elements $\left\{L_{w}\right\}_{w>0}$ satisfy the further condition that $L_{w} \in L_{\mu_{G}}^{1}(G)$ for every $w>0$. We then put $\gamma_{w}:=\int_{G} L_{w}(z) d \mu_{G}(z)$ for $w>0$, so $\left\{\gamma_{w}\right\}_{w>0}$ is a bounded net.

It is clear that if in particular we take $\mu_{w}^{s}(A)=\int_{A} L_{w}\left(s-h_{w}(t)\right) d \mu_{H}(t)$, $A \in \mathcal{B}(H), s \in G, w>0$, then the family $\left\{\mu_{w}\right\}_{w>0}$ is regular. Indeed, condition (a) of regularity is obviously satisfied and (b) is a consequence of (L2) and (L3). Finally assumption (c) is satisfied with $\eta_{w}=\gamma_{w}, w>0$.

Denote by $\eta_{H}$ a modular on $X(H)$. Given $E>0$ let

$$
\mathcal{L}_{E} \equiv \mathcal{L}\left(G, H,\left\{h_{w}\right\},\left\{\gamma_{w}\right\}, E\right)
$$


be the subset of $L_{\eta_{G}}(G)$ whose elements $f$ satisfy the following assumption (see $[7,28])$ :

$$
\limsup _{w \rightarrow \infty} \gamma_{w} \eta_{H}\left[\lambda\left(f \circ h_{w}\right)\right] \leq E \eta_{G}[\lambda f]
$$

for every $\lambda>0$. Then we may establish the following:

Theorem 3. Let $\left\{K_{w}\right\}_{w>0} \in \mathcal{K},\left\{L_{w}\right\}_{w>0} \in \mathcal{L}^{*}$, let $\varrho_{G}$ be a monotone modular and suppose that the triple $\left\{\varrho_{H}, \psi, \eta_{H}\right\}$ is properly directed; assume moreover that the family $\mu_{w}^{s}(A)=\int_{A} L_{w}\left(s-h_{w}(t)\right) d \mu_{H}(t), A \in \mathcal{B}(H)$, $s \in G$, is compatible with the couple $\left(\varrho_{G}, \varrho_{H}\right)$. Then, given any two functions $f$ and $g$ in the domain of all operators $T_{w}$ for $w>0$, such that $f-g \in \mathcal{L}_{E}$ for some $E>0$, there is an absolute constant $P>0$, depending on $E>0$, such that for any $\lambda \in] 0,1[$ there exists a constant $c>0$ satisfying

$$
\limsup _{w \rightarrow \infty} \varrho_{G}\left[c\left(T_{w} f-T_{w} g\right)\right] \leq P \eta_{G}[\lambda(f-g)] .
$$

Proof. Let $\lambda>0$ be fixed with $\lambda<1$. By the $\left(L_{w}, \psi\right)$-Lipschitz condition,

$$
\begin{aligned}
\varrho_{G}\left[c\left(T_{w} f-T_{w} g\right)\right] \\
\quad=\varrho_{G}\left[c \int_{H}\left[K_{w}\left(\cdot-h_{w}(t), f\left(h_{w}(t)\right)\right)-K_{w}\left(\cdot-h_{w}(t), g\left(h_{w}(t)\right)\right)\right] d \mu_{H}(t)\right] \\
\leq \varrho_{G}\left[c \int_{H}\left|K_{w}\left(\cdot-h_{w}(t), f\left(h_{w}(t)\right)\right)-K_{w}\left(\cdot-h_{w}(t), g\left(h_{w}(t)\right)\right)\right| d \mu_{H}(t)\right] \\
\leq \varrho_{G}\left[c \int_{H} L_{w}\left(\cdot-h_{w}(t)\right) \psi\left(\left|f\left(h_{w}(t)\right)-g\left(h_{w}(t)\right)\right|\right) d \mu_{H}(t)\right] .
\end{aligned}
$$

By regularity of $\left\{\mu_{w}^{s}\right\}$ defined above with $\eta_{w}=\gamma_{w}=\left\|L_{w}\right\|_{L^{1}(G)}$, and by compatibility with the couple $\left(\varrho_{G}, \varrho_{H}\right)$, we have, for sufficiently large $w>0$,

$$
\varrho_{G}\left[c\left(T_{w} f-T_{w} g\right)\right] \leq M \gamma_{w} \varrho_{H}\left[c D \psi\left(\left|(f-g) \circ h_{w}\right|\right)\right]+b_{w} .
$$

Then, since $\left\{\varrho_{H}, \psi, \eta_{H}\right\}$ is properly directed, for $c D \leq C_{\lambda}$ we obtain

$$
\begin{aligned}
\varrho_{G}\left[c\left(T_{w} f-T_{w} g\right)\right] & \leq M \gamma_{w} \varrho_{H}\left[C_{\lambda} \psi\left(\left|(f-g) \circ h_{w}\right|\right)\right]+b_{w} \\
& \leq M \gamma_{w} \eta_{H}\left[\lambda\left((f-g) \circ h_{w}\right)\right]+b_{w} .
\end{aligned}
$$

Since $f-g \in \mathcal{L}_{E}$, we have

$$
\limsup _{w \rightarrow \infty} \varrho_{G}\left[c\left(T_{w} f-T_{w} g\right)\right] \leq M E \eta_{G}[\lambda(f-g)] .
$$

The assertion follows easily by putting $P=M E$.

Now we are ready to prove the main theorem of this section:

Theorem 4. Let $\left\{K_{w}\right\}_{w>0} \in \mathcal{K},\left\{L_{w}\right\}_{w>0} \in \mathcal{L}^{*}$ and let $\varrho_{G}, \eta_{G}$ be monotone, absolutely and strongly finite and absolutely continuous modulars, and $\varrho_{H}$ be a modular such that $\left\{\varrho_{H}, \psi, \eta_{H}\right\}$ is properly directed. Suppose that the family $\mu_{w}^{s}(A)=\int_{A} L_{w}\left(s-h_{w}(t)\right) d \mu_{H}(t), A \in \mathcal{B}(H), s \in G, w>0$, is 
compatible with the couple $\left(\varrho_{G}, \varrho_{H}\right)$. Then for every $f \in L_{\varrho+\eta}(G)$ such that $f-C_{\mathrm{c}}(G) \subset \mathcal{L}_{E}$ for some $E>0$, there is a constant $c>0$ such that

$$
\lim _{w \rightarrow \infty} \varrho_{G}\left[c\left(T_{w} f-f\right)\right]=0 .
$$

Proof. Let $f$ be as in the statement. By the density theorem, there is a $\bar{\lambda}>0$ (we may take $\bar{\lambda}<1$ ) and a sequence $\left\{f_{n}\right\} \subset C_{\mathrm{c}}(G)$ such that

$$
\left(\varrho_{G}+\eta_{G}\right)\left[\bar{\lambda}\left(f_{n}-f\right)\right] \rightarrow 0, \quad n \rightarrow \infty .
$$

Fix $\varepsilon>0$ and an integer $\widetilde{n}$ such that for every $n \geq \widetilde{n}$,

$$
\left(\varrho_{G}+\eta_{G}\right)\left[\bar{\lambda}\left(f_{n}-f\right)\right]<\varepsilon .
$$

Choose a constant $c>0$ such that $c \leq \min \left\{C_{\bar{\lambda}} /(3 D), \bar{\lambda} / 3\right\}$. Then

$$
\begin{aligned}
& \varrho_{G}\left[c\left(T_{w} f-f\right)\right] \\
& \quad \leq \varrho_{G}\left[3 c\left(T_{w} f-T_{w} f_{\widetilde{n}}\right)\right]+\varrho_{G}\left[3 c\left(T_{w} f_{\widetilde{n}}-f_{\widetilde{n}}\right)\right]+\varrho_{G}\left[3 c\left(f_{\widetilde{n}}-f\right)\right] .
\end{aligned}
$$

Now, applying Theorem 3 to the first summand, we obtain

$$
\limsup _{w \rightarrow \infty} \varrho_{G}\left[3 c\left(T_{w} f-T_{w} f_{\widetilde{n}}\right)\right] \leq P \eta_{G}\left[\bar{\lambda}\left(f-f_{\widetilde{n}}\right)\right],
$$

where, without loss of generality, we can suppose $P>1$. Since by Theorem 2 we have $\lim _{w \rightarrow \infty} \varrho_{G}\left[3 c\left(T_{w} f_{\widetilde{n}}-f_{\widetilde{n}}\right)\right]=0$, applying (1), we obtain

$$
\limsup _{w \rightarrow \infty} \varrho_{G}\left[c\left(T_{w} f-f\right)\right] \leq P\left(\varrho_{G}+\eta_{G}\right)\left(\bar{\lambda}\left(f-f_{\widetilde{n}}\right)\right) \leq P \varepsilon ;
$$

hence the assertion follows from the arbitrariness of $\varepsilon>0$.

4. Applications. For the sake of simplicity, in this section we consider the particular case of Musielak-Orlicz spaces, where we take $H \subset G$ ( $H$ is a subgroup of $G$ ) and $\mu_{H}$ is the Haar measure of the subgroup $H$.

Let $\Phi$ be the class of all functions $\varphi: G \times \mathbb{R}_{0}^{+} \rightarrow \mathbb{R}_{0}^{+}$which satisfy the conditions:

1) $\varphi(\cdot, u)$ is measurable on $G$ for every $u \in \mathbb{R}_{0}^{+}$;

2) for every $s \in G, \varphi(s, \cdot)$ is continuous and nondecreasing on $\mathbb{R}_{0}^{+}$with $\varphi(s, 0)=0$ and $\varphi(s, u)>0$ for $u>0$;

3) $\varphi$ is $\tau$-bounded, i.e. there are a constant $C \geq 1$ and a globally measurable function $F: G \times G \rightarrow \mathbb{R}_{0}^{+}$such that for every $t, s \in G$ and $u \geq 0$,

$$
\varphi(s, u) \leq \varphi(t, C u)+F(t, s) .
$$

If moreover $\varphi(s, \cdot)$ is also convex on $u \in \mathbb{R}_{0}^{+}$, for every $s \in G$, then we will write $\varphi \in \widetilde{\Phi}$.

Let $\left\{\mu_{w}^{s}\right\}_{w>0}$ be the family defined by $\mu_{w}^{s}(A)=\int_{A} L_{w}\left(s-h_{w}(t)\right) d \mu_{H}(t)$, $A \in \mathcal{B}(H),\left\{L_{w}\right\} \in \mathcal{L}, s \in G$ and $w>0$, which, as seen before, is regular. 
We put

$$
\varrho_{G}(f)=\int_{G} \varphi(s,|f(s)|) d \mu_{G}(s), \quad \varrho_{H}(g)=\int_{H} \varphi(t,|g(t)|) d \mu_{H}(t)
$$

for $f \in L_{\varrho}(G)$ and $g \in L_{\varrho}(H)$ where $L_{\varrho}(G)$ and $L_{\varrho}(H)$ are the corresponding Musielak-Orlicz spaces, also denoted by $L^{\varphi}(G)$ and $L^{\varphi}(H)$ respectively (see $[23,21,26])$. Finally we put

$$
\delta_{w}=\iint_{G H} F(t, s) L_{w}\left(s-h_{w}(t)\right) d \mu_{H}(t) d \mu_{G}(s) .
$$

In [22] it is proved that if $\delta_{w} \rightarrow 0$ as $w \rightarrow \infty$, then the family $\left\{\mu_{w}^{s}\right\}_{w>0}$ is compatible with the couple $\left(\varrho_{G}, \varrho_{H}\right)$, and this happens if $\varphi$ is a $\tau$-bounded function in the strong sense, which means that $\|F(\cdot, s)\|_{L^{1}(\mathbb{R})} \rightarrow 0$ as $s \rightarrow 0$, where $F$ is the function from condition 3).

Now let $\varrho_{H}$ and $\eta_{H}$ be two modulars on $X(H)$ defined by

$$
\varrho_{H}(f)=\int_{H} \varphi(t,|f(t)|) d \mu_{H}(t), \quad \eta_{H}(f)=\int_{H} \xi(t,|f(t)|) d \mu_{H}(t),
$$

for $\varphi \in \widetilde{\Phi}, \xi \in \Phi$. In this case we see that the triple $\left\{\varrho_{H}, \psi, \eta_{H}\right\}$, where $\psi \in \Psi$, is properly directed if for every $\lambda \in] 0,1\left[\right.$ there exists a $\left.C_{\lambda} \in\right] 0,1[$ satisfying

$$
\varphi\left(t, C_{\lambda} \psi(u)\right) \leq \xi(t, \lambda u)
$$

for every $t \in H, u \in \mathbb{R}_{0}^{+}$(see $[6,28]$ in the case of Orlicz spaces). Moreover it is clear that the modular generating a Musielak-Orlicz space is monotone, absolutely continuous, absolutely finite and strongly finite if $\varphi$ is locally integrable, i.e. $\int_{A} \varphi(s, u) d \mu_{G}(s)<\infty$ for every $u>0$ and every measurable subset $A$ of $G$ with $\mu_{G}(A)<\infty$ (see [24]). Moreover, in the case of Orlicz spaces, the above properties of the modular are always satisfied.

Now we consider some interesting examples of operators $T_{w}$.

I) Convolution integral operators. Let $G=H=\left(\mathbb{R}^{N},+\right)$ and $\mu_{G}=$ $\mu_{H}=d t$, the Lebesgue measure. Let

$$
\varrho_{G}(f)=\varrho_{H}(f)=\int_{\mathbb{R}^{N}} \varphi(t,|f(t)|) d t, \quad \eta_{G}(f)=\eta_{H}(f)=\int_{\mathbb{R}^{N}} \xi(t,|f(t)|) d t
$$

with $\varphi \in \widetilde{\Phi}, \xi \in \Phi$ and let $L^{\varphi}\left(\mathbb{R}^{N}\right), L^{\xi}\left(\mathbb{R}^{N}\right)$ be the Musielak-Orlicz spaces generated by the modulars $\varrho$ and $\eta$. Put $h_{w}(t)=t$ for every $w>0$. Then we obtain

$$
\left(T_{w} f\right)(s)=\int_{\mathbb{R}^{N}} K_{w}(s-t, f(t)) d t, \quad s \in \mathbb{R}^{N} .
$$

In this case it is clear that $\mathcal{L}_{E}=L^{\xi}\left(\mathbb{R}^{N}\right)$ with $E=\sup _{w} \gamma_{w}<\infty$. Moreover, it is easy to see that in the linear case, i.e. when $K_{w}(s, u)=K_{w}(s) u$, the 
assumptions ( $\mathrm{L} i)$ and $(\mathrm{K} i), i=1,2,3$, become the classical ones for approximate identities with compact support (see [7, 28]). So our theory includes, as particular cases, the classical convergence theorems in Musielak-Orlicz spaces, in Orlicz spaces and in $L^{p}$-spaces with $p \geq 1$ (see $[15,23]$ ) for linear integral operators of convolution type.

II) Mellin convolution operators. Analogous applications can be deduced for Mellin convolution operators of the form

$$
\left(T_{w} f\right)(s) \equiv(\mathcal{M} f)(s)=\int_{0}^{\infty} K_{w}\left(s t^{-1}, f(t)\right) t^{-1} d t, \quad s>0,
$$

where $\left\{K_{w}\right\}_{w>0}$ is a suitable kernel. Here we take $G=H=\left(\mathbb{R}^{+}, \cdot\right)$, $\mu_{G}=\mu_{H}=\int t^{-1} d t, h_{w}(t)=t$ for every $w>0, \varrho_{G}(f)=\varrho_{H}(f)=$ $\int_{0}^{\infty} \varphi(t,|f(t)|) t^{-1} d t, \eta_{G}(f)=\eta_{H}(f)=\int_{0}^{\infty} \xi(t,|f(t)|) t^{-1} d t$ with $\varphi \in \widetilde{\Phi}$, $\xi \in \Phi$, and $L^{\varphi}\left(\mathbb{R}^{+}\right), L^{\xi}\left(\mathbb{R}^{+}\right)$are Musielak-Orlicz spaces. Also in this case we have $\mathcal{L}_{E} \equiv L^{\xi}\left(\mathbb{R}^{+}\right)$for the same $E$ as before. The above operators are connected with the theory of moment type operators (see [1, 2]), as well as with the theory of the Mellin transform (see [13, 14, 3, 9]). Moreover in the case of $G=H=\left(\mathbb{R}_{+}^{n}, \bullet\right)$, where $\mathbb{R}_{+}^{n}=(] 0, \infty[)^{n}$ and "•" is defined by $s \bullet t=\left(s_{1} t_{1}, \ldots, s_{n} t_{n}\right) \in \mathbb{R}_{+}^{n}$, for $s=\left(s_{1}, \ldots, s_{n}\right), t=\left(t_{1}, \ldots, t_{n}\right) \in \mathbb{R}_{+}^{n}$ the previous theory also includes the multidimensional version of nonlinear Mellin convolution operators (see [28]). In fact, $G$ is a locally compact, topological, abelian group with neutral element $\theta=1=(1, \ldots, 1)$, the inverse of $t$ is given by $t^{-1}=\left(t_{1}^{-1}, \ldots, t_{n}^{-1}\right)$ and if we put $\langle t\rangle=\prod_{k=1}^{n} t_{k}$, the Haar measure $\mu_{G}$ is given by

$$
\mu_{G}=\mu_{H}=\int \frac{d t}{\langle t\rangle}
$$

$d t$ being the Lebesgue measure.

III) Generalized sampling operators. Let $G=(\mathbb{R},+), H=(\mathbb{Z},+)$ with $\mu_{G}$ the Lebesgue measure on $\mathbb{R}, \mu_{H}$ the counting measure on $\mathbb{Z}$ and take $h_{w}: \mathbb{Z} \rightarrow \mathbb{R}$ of the form $h_{w}(k)=k / w, k \in \mathbb{Z}, w>0$. In this case we obtain the nonlinear version of the generalized sampling operators of the form:

$$
\left(T_{w} f\right)(s)=\sum_{k=-\infty}^{\infty} K(w s-k, f(k / w)), \quad s \in \mathbb{R}, w>0 .
$$

Moreover,

$$
\begin{array}{ll}
\varrho_{G}(f)=\int_{\mathbb{R}} \varphi(s,|f(s)|) d s, & \varrho_{H}(g)=\sum_{k=-\infty}^{\infty} \varphi(k,|g(k)|), \\
\eta_{G}(f)=\int_{\mathbb{R}} \xi(s,|f(s)|) d s, & \eta_{H}(g)=\sum_{k=-\infty}^{\infty} \xi(k,|g(k)|),
\end{array}
$$


with $\varphi \in \widetilde{\Phi}$ and $\xi \in \Phi$, are the modulars generating respectively the Musielak-Orlicz spaces $L^{\varphi}(\mathbb{R}), L^{\varphi}(\mathbb{Z})$ and $L^{\xi}(\mathbb{R}), L^{\xi}(\mathbb{Z})$. Let $L_{w}(s)=L(w s)$ for $s \in \mathbb{R}, w>0$, where $L \in L^{1}(\mathbb{R})$ has compact support.

It is proved (see [22]) that if $\|F(\cdot, s)\|_{l^{1}(\mathbb{Z})}$ is uniformly bounded with respect to $s \in \mathbb{R}$, then $\delta_{w} \rightarrow 0$ as $w \rightarrow \infty$ and hence the family $\mu_{w}^{s}(H)=$ $\int_{H} L_{w}\left(s-h_{w}(t)\right) d \mu_{H}(t), s \in \mathbb{R}, w>0$, is compatible with the couple $\left(\varrho_{G}, \varrho_{H}\right)$ defined above.

From now on we suppose that $\varphi$ and $\xi$ are locally integrable and that $\|F(\cdot, s)\|_{l^{1}(\mathbb{Z})}$ is uniformly bounded with respect to $s \in \mathbb{R}$. In this case, for $K_{w}(s, \cdot)=K(w s, \cdot), w>0, s \in \mathbb{R}$, assumption (K3) becomes

$$
r_{n}^{w}(s):=\sup _{1 / n \leq|u| \leq n}\left|\frac{1}{u} \sum_{k=-\infty}^{\infty} K(w s-k, u)-1\right| \rightarrow 0 \quad \text { as } w \rightarrow \infty
$$

a.e. $s \in \mathbb{R}$, while (L2) becomes

$$
\sum_{k=-\infty}^{\infty} L(w s-k) \leq N
$$

for some constant $N>0$ and for every $s \in \mathbb{R}, w>0$; in a similar manner we rephrase (L3). Moreover assumption (L1) is always satisfied since $L_{w}(z)=$ $L(w z)$ and $L$ has compact support in $\mathbb{R}$, and

$$
\int_{\mathbb{R}} L_{w}(z) d z=\int_{\mathbb{R}} L(w z) d z=\frac{\|L\|_{L^{1}(\mathbb{R})}}{w}=\gamma_{w} .
$$

Now in the particular case of $K_{w}$ linear, i.e. $K_{w}(s, u)=K_{w}(s) u$, we have $L_{w}(s) \equiv\left|K_{w}(s)\right|$ (for the linear case see $[11,27]$ ). If in this case we suppose that:

(i) $\sum_{k \in \mathbb{Z}} K(s-k)=1$ for every $s \in \mathbb{R}$,

(ii) $\sup _{s \in \mathbb{R}} \sum_{k \in \mathbb{Z}} L(s-k)<\infty$,

then $(\alpha)$ and $(\beta)$ are satisfied. Assumptions (i) and (ii) are very common in the theory of sampling series.

The condition $(+)$ of the class $\mathcal{L}_{E}$ now becomes

$$
\limsup _{w \rightarrow \infty} \frac{1}{w} \sum_{k=-\infty}^{\infty} \xi(k, \lambda|f(k / w)|) \leq S \int_{-\infty}^{\infty} \xi(s, \lambda|f(s)|) d s
$$

for every $\lambda>0$ and for some constant $S>0$. As mentioned in [7, 28], the density result (Theorem 1) can be restated in a stronger form with $C_{\mathrm{c}}^{\infty}(\mathbb{R})$ instead of $C_{\mathrm{c}}(\mathbb{R})$ (see [17]). So we may formulate, as application of Theorem 4, the following corollary: 
Corollary 2. Let $\varphi \in \widetilde{\Phi}, \xi \in \Phi$ and $f \in L^{\varphi+\xi}(\mathbb{R})$. Suppose that $\left\{\varrho_{H}, \psi, \eta_{H}\right\}$ is properly directed. Moreover let $f: \mathbb{R} \rightarrow \mathbb{R}$ be such that

$$
\limsup _{w \rightarrow \infty} \frac{1}{w} \sum_{k=-\infty}^{\infty} \xi(k, \lambda|g(k / w)|) \leq S \int_{-\infty}^{\infty} \xi(s, \lambda|g(s)|) d s
$$

for every $g \in f-C_{\mathrm{c}}^{\infty}(\mathbb{R}), \lambda>0$ and for some constant $S>0$. Then there exists a constant $c>0$ such that

$$
\lim _{w \rightarrow \infty} \int_{-\infty}^{\infty} \varphi\left(s, c\left|\sum_{k=-\infty}^{\infty} K(w s-k, f(k / w))-f(s)\right|\right) d s=0 .
$$

As an example, we consider a function $\xi: \mathbb{R} \times \mathbb{R}_{0}^{+} \rightarrow \mathbb{R}_{0}^{+}$of the form

$$
\xi(s, u)=\widetilde{\xi}(s) \gamma(u)
$$

where $\widetilde{\xi}: \mathbb{R} \rightarrow \mathbb{R}_{0}^{+}$satisfies the following conditions:

1) $\widetilde{\xi}$ is measurable;

2) there exists $a>0$ such that $\widetilde{\xi}(s) \geq a$ for every $s \in \mathbb{R}$;

3) the sequence $\varepsilon_{k}=\widetilde{\xi}(k), k \in \mathbb{Z}$, is bounded.

We also suppose that $\gamma$ is a continuous nondecreasing function such that $\gamma(0)=0$ and $\gamma(u)>0$ for every $u>0$. In this case, for any function $f$, we have

$$
\text { (•) } \begin{aligned}
\frac{1}{w} \sum_{k=-\infty}^{\infty} \xi(k, \lambda|f(k / w)|) & =\frac{1}{w} \sum_{k=-\infty}^{\infty} \widetilde{\xi}(k / w) \gamma(\lambda|f(k / w)|) \frac{\widetilde{\xi}(k)}{\widetilde{\xi}(k / w)} \\
& \leq \frac{\Gamma}{w} \sum_{k=-\infty}^{\infty} \xi(k / w, \lambda|f(k / w)|)
\end{aligned}
$$

where $\Gamma=\|\widetilde{\xi}(k)\|_{l^{\infty}} / a$ is a positive constant. The last term of inequality $(\bullet)$ represents the Riemann sum of $\xi(s, \lambda|f(s)|)$.

Thus, if $f: \mathbb{R} \rightarrow \mathbb{R}$ and $g \in f-C_{\mathrm{c}}^{\infty}(\mathbb{R})$ are such that the function $\xi(s, \lambda|g(s)|)$ is Riemann integrable and of bounded variation on $\mathbb{R}$, then $g \in \mathcal{L}_{E}$ (see [18]). Thus we have:

Corollary 3. Let the function $\xi: \mathbb{R} \times \mathbb{R}_{0}^{+} \rightarrow \mathbb{R}_{0}^{+}$of the form $\xi(s, u)=$ $\widetilde{\xi}(s) \gamma(u)$ satisfy the above conditions and let $\left\{\varrho_{H}, \psi, \eta_{H}\right\}$ be properly directed. If $f: \mathbb{R} \rightarrow \mathbb{R}$ is such that $f \in L^{\varphi+\xi}(\mathbb{R})$ and, for some $g \in f-C_{\mathrm{c}}^{\infty}(\mathbb{R})$, $\xi(s, \lambda|g(s)|)$ is a Riemann integrable function of bounded variation on $\mathbb{R}$ for every $\lambda>0$, then there exists a constant $c>0$ such that

$$
\lim _{w \rightarrow \infty} \int_{-\infty}^{\infty} \varphi\left(s, c\left|\sum_{k=-\infty}^{\infty} K(w s-k, f(k / w))-f(s)\right|\right) d s=0 .
$$


In the particular case of Orlicz spaces, it is possible to give sufficient conditions on the function $f: \mathbb{R} \rightarrow \mathbb{R}$ in order that $\xi(\lambda|f(s)|)$ is a Riemann integrable function of bounded variation on $\mathbb{R}$, for every $\lambda>0$; namely it suffices to assume that $f$ is an absolutely Riemann integrable function on $\mathbb{R}$ with bounded variation on $\mathbb{R}$ and that $\xi: \mathbb{R}_{0}^{+} \rightarrow \mathbb{R}_{0}^{+}$is locally Lipschitz on $\mathbb{R}_{0}^{+}$(see Corollary 2 of [28]).

Remarks 2. (a) The previous theory also contains the case of the multivariate sampling series of a function $f: \mathbb{R}^{N} \rightarrow \mathbb{R}$, in its nonlinear form. Indeed, it suffices to take $G=\left(\mathbb{R}^{N},+\right), H=\left(\mathbb{Z}^{N},+\right)$ with the Lebesgue measure and the counting measure respectively. Then the above generalized sampling operators take the form

$$
\left(T_{w} f\right)(s)=\sum_{k \in \mathbb{Z}^{N}} K(w s-k, f(k / w))
$$

for $s \in \mathbb{R}^{N}, w \in \mathbb{R}_{+}^{N}, K: \mathbb{R}^{N} \times \mathbb{R} \rightarrow \mathbb{R}$ and $f: \mathbb{R}^{N} \rightarrow \mathbb{R}$.

In the linear case, the multivariate generalized sampling series have been studied in $[12,7]$. Here $w$ is a vector, i.e. $w=\left(w_{1}, \ldots, w_{N}\right) \in \mathbb{R}_{+}^{N}$, and we define $w_{1} \leq w_{2}$ if and only if $w_{1}^{i} \leq w_{2}^{i}$ for $i=1, \ldots, N$. Moreover if $w=\left(w_{1}, \ldots, w_{N}\right), s=\left(s_{1}, \ldots, s_{N}\right), k=\left(k_{1}, \ldots, k_{N}\right)$, we set $w s=$ $\left(w_{1} s_{1}, \ldots, w_{N} s_{N}\right), k / w=\left(k_{1} / w_{1}, \ldots, k_{N} / w_{N}\right)$, and $w \rightarrow \infty$ means that $w_{i} \rightarrow \infty$ for each $i=1, \ldots, N$.

Moreover we have

$$
\int_{\mathbb{R}^{N}} L_{w}(z) d z=\int_{\mathbb{R}^{N}} L(w z) d z=\frac{1}{\prod_{k=1}^{N} w_{k}} \int_{\mathbb{R}^{N}} L(z) d z=\frac{\|L\|_{L^{1}\left(\mathbb{R}^{N}\right)}}{\prod_{k=1}^{N} w_{k}}=\gamma_{w}
$$

and assumption $(+)$ of the class $\mathcal{L}_{E}$ becomes

$$
\limsup _{w \rightarrow \infty} \frac{1}{\prod_{k=1}^{N} w_{k}} \sum_{k \in \mathbb{Z}^{N}} \xi\left(k, \lambda \mid f(k / w \mid) \leq S \int_{\mathbb{R}^{N}} \xi(s, \lambda|f(s)|) d s\right.
$$

for every $\lambda>0$ and some constant $S>0$.

(b) In the previous theory we may replace the real parameter $w>0$ by an abstract parameter $w$ varying in an arbitrary filtering partially ordered set $\mathcal{W}$.

(c) In the case of $\varphi(u)=u^{p}, p \geq 1$, Corollaries 2 and 3 give strong convergence results in $L^{p}(\mathbb{R})$ for the nonlinear sampling series of $f$.

\section{References}

[1] F. Barbieri, Approssimazione mediante nuclei momento, Atti Sem. Mat. Fis. Univ. Modena 32 (1983), 308-328.

[2] C. Bardaro, On approximation properties for some classes of linear operators of convolution type, ibid. 33 (1984), 329-356. 
[3] C. Bardaro and I. Mantellini, Linear integral operators with homogeneous kernel: approximation properties in modular spaces. Applications to Mellin-type convolution operators and to some classes of fractional operators, Applied Math. Rev. Vol. I, G. Anastassiou (ed.), World Sci., 2000, 45-67.

[4] - - - On approximation properties of Urysohn integral operators, Internat. J. Pure Appl. Math. (2002), to appear.

[5] C. Bardaro, J. Musielak and G. Vinti, Approximation by nonlinear integral operators in some modular function spaces, Ann. Polon. Math. 63 (1996), 173-182.

[6] C. Bardaro and G. Vinti, Modular approximation by nonlinear integral operators on locally compact groups, Comment. Math. 35 (1995), 25-47.

[7] —, - A general approach to the convergence theorems of generalized sampling series, Appl. Anal. 64 (1997), 203-217.

[8] - - - Uniform convergence and rate of approximation for a nonlinear version of the generalized sampling operator, Results in Math., special issue dedicated to P. L. Butzer, 34 (1998), 224-240.

[9] - - - Urysohn integral operators with homogeneous kernels: approximation properties in modular spaces, Comment. Math. 42 (2002), 145-182.

[10] L. Bezuglaya and V. Katsnel'son, The sampling theorem for functions with limited multi-band spectrum I, Z. Anal. Anwendungen 12 (1993), 511-534.

[11] P. L. Butzer, A survey of the Whittaker-Shannon sampling theorem and some of its extensions, J. Math. Res. Exposition 3 (1983), 185-212.

[12] P. L. Butzer, A. Fischer and R. L. Stens, Generalized sampling approximation of multivariate signals; theory and some applications, Note Mat. 10, Suppl. no. 1, (1990), 173-191.

[13] P. L. Butzer and S. Jansche, The exponential sampling theorem of signal analysis, Atti Sem. Mat. Fis. Univ. Modena Suppl. Vol. 46, a special volume dedicated to Prof. C. Vinti, 1998, 99-122.

[14] - - - Mellin-Fourier series and the classical Mellin transform, Comput. Math. Appl., to appear.

[15] P. L. Butzer and R. J. Nessel, Fourier Analysis and Approximation I, Academic Press, New York, 1971.

[16] P. L. Butzer and R. L. Stens, Linear prediction by samples from the past, in: Advanced Topics in Shannon Sampling and Interpolation Theory, R. J. Marks II (ed.), Springer, New York, 1993, 157-183.

[17] G. B. Folland, Real Analysis: Modern Techniques and Their Applications, Wiley, 1984.

[18] S. Haber and O. Shisha, Improper integral, simple integrals and numerical quadrature, J. Approx. Theory 11 (1974), 1-15.

[19] E. Hewitt and K. A. Ross, Abstract Harmonic Analysis, Springer, Berlin, 1963.

[20] J. R. Higgins, Sampling Theory in Fourier and Signal Analysis: Foundations, Oxford Univ. Press, Oxford, 1996.

[21] W. M. Kozłowski, Modular Function Spaces, Pure Appl. Math., Dekker, New York, 1988.

[22] I. Mantellini, Generalized sampling operators in modular spaces, Comment. Math. 38 (1998), 77-92.

[23] J. Musielak, Orlicz Spaces and Modular Spaces, Lecture Notes in Math. 1034, Springer, 1983.

[24] -, Nonlinear approximation in some modular function spaces I, Math. Japon. 38 (1993), 83-90.

[25] J. Musielak and W. Orlicz, On modular spaces, Studia Math. 28 (1959), 49-65. 
[26] M. M. Rao and Z. D. Ren, Theory of Orlicz Spaces, Monographs Textbooks Pure Appl. Math. 146, Dekker, New York, 1991.

[27] S. Ries and R. L. Stens, Approximation by generalized sampling series, in: Constructive Theory of Functions '84, Sofia, 1984, 746-756.

[28] G. Vinti, A general approximation result for nonlinear integral operators and applications to signal processing, Appl. Anal. 79 (2001), 217-238.

Department of Mathematics and Informatics

University of Perugia

Via Vanvitelli 1

06123 Perugia, Italy

E-mail: mantell@dipmat.unipg.it mategian@unipg.it

Web: http://www.unipg.it/ ${ }^{2}$ mategian

Reçu par la Rédaction le 1.2.2002

Révisé le 10.5.2002 